\title{
Preclinical immunopharmacologic assessment of KPL-404, a novel, humanized, non-depleting antagonistic anti-CD40 monoclonal antibody
}

Authors: Sujatha Muralidharan, Moses Njenga, Tracy Garron, Kent Bondensgaard, John F. Paolini

Affiliations: Kiniksa Pharmaceuticals Corp. 


\section{Running title: Immunopharmacologic assessment of KPL-404, a CD40 antagonist}

\section{Address for Correspondence:}

Name, degree: $\quad$ John F. Paolini, M.D, Ph.D.

Affiliation: Kiniksa Pharmaceuticals Corp.

Address: 100 Hayden Avenue

City, State ZIP: $\quad$ Lexington, MA, 02421

Tel: $\quad 617-386-6479$

Email: $\quad$ jpaolini@kiniksa.com

Number of text pages: 26

Number of tables: 1

Number of figures: 7

Number of references: 30

Number of words in abstract: $240 / 250$

Number of words in introduction: 683/750

Number of words in discussion: 1401/1500

Abbreviations: ADA, anti-drug antibody; ADCC, Antibody Dependent Cellular Cytotoxicity; CDC, Complement Dependent Cytotoxicity; ECLIA, Electrochemiluminescent Immunoassay; ELISA, EnzymeLinked Immunosorbent Assay; Fc, fragment crystallizable; IV, intravenous; ITP, immune thrombocytopenic purpura; KLH, Keyhole limpet hemocyanin; MAPK, mitogen-activated protein kinase; MFI, mean fluorescence intensity; MRD, minimum required dilution; NF- $\kappa \mathrm{B}$, nuclear factor $\kappa \mathrm{B}$; NK, natural killer; PBMC, peripheral blood mononuclear cell; PI3K, phosphoinositide 3-kinase; PK/PD, pharmacokinetic/pharmacodynamic; RA, rheumatoid arthritis; RO, receptor occupancy; SLE, systemic lupus erythematosus; SPR, surface plasmon resonance; TDAR, T-cell Dependent Antibody Response; TMDD, target-mediated drug disposition; TNF, tumor necrosis factor; TRAF, TNFR-associated factors; TT, tetanus toxoid;

Recommended section: Inflammation, Immunopharmacology, and Asthma 


\section{$\underline{\text { ABSTRACT }}$}

The CD40/CD40L pathway plays a major role in multiple inflammatory processes involving different immune and stromal cells. Abnormal activation of this pathway has been implicated in pathogenesis of complex autoimmune diseases including SLE, RA, Graves' disease, and Sjogren's Syndrome. We completed in vitro and in vivo preclinical characterization of KPL-404, a novel humanized anti-CD40 IgG4 monoclonal antibody, in order to demonstrate its potency, efficacy, and pharmacokinetic profile; safety was also assessed. In vitro, KPL-404 bound recombinant human and cynomolgus monkey CD40 with comparable affinity in the nanomolar range. KPL-404 binding to cell surface CD40 did not induce antibody- or complement-mediated cytotoxicity of CD40-expressing cells. Pharmacological antagonistic activity of KPL-404 was demonstrated in vitro by inhibition of CD40-mediated downstream NF-kB activation. In the in vivo study with cynomolgus monkeys, KPL-404, administered intravenously as a single dose $(10 \mathrm{mg} / \mathrm{kg})$ or two monthly doses of 1 or $5 \mathrm{mg} / \mathrm{kg}$, did not elicit observable safety findings, including thrombocytopenia over 8 weeks. KPL-404 engaged CD40 expressed on peripheral B cells for 2 and 4 weeks after a single administration of 5 or $10 \mathrm{mg} / \mathrm{kg} \mathrm{IV}$, respectively, without depletion of peripheral B cells. At $5 \mathrm{mg} / \mathrm{kg} \mathrm{IV}$, KPL-404 blocked both primary and secondary responses to T-cell dependent antibody responses to test antigens, KLH and TT. These data illustrated the relationship between KPL-404 serum concentration and in pharmacodynamic effects of CD40-targeting circulation and in lymphoid tissues. These data support clinical development of KPL-404 in autoimmune diseases. 


\section{SIGNIFICANCE STATEMENT}

We aimed to develop a potent and efficacious CD40 antagonist. In vitro and in vivo findings characterize

KPL-404 as a blocking anti-CD40 antibody that potently inhibits primary and secondary antibody

responses at pharmacologically relevant concentrations with a favorable pharmacokinetic profile and did

not deplete B-cells by antibody dependent cellular cytotoxicity or apoptosis ("non-depleting"). These

findings support clinical development of KPL-404 as a potential therapeutic in autoimmune diseases.

\section{KEYWORDS}

CD40; CD40L; costimulation; immunosuppression; autoimmune disease; pharmacology 


\section{INTRODUCTION}

The CD40/CD40L pathway mediates multiple inflammatory processes by activating a number of immune and stromal cell populations (Karnell et al., 2019b). The ligation of CD40 with CD40L activates antigen-presenting cells to promote $\mathrm{T}$ cell activation and $\mathrm{T}$ cell-dependent humoral responses, including $\mathrm{B}$ cell differentiation and antibody production (Danese et al., 2004; Hernandez et al., 2007; Inwald et al., 2003; Karnell et al., 2019b; Phipps, 2008). Engagement of the CD40/CD40L pathway additionally drives the activation of other cell subsets including macrophages, neutrophils, mast cells, stromal fibroblasts, and chondrocytes (Karnell et al., 2019b). Dysregulation of the CD40/CD40L pathway has been associated with a variety of autoimmune conditions where abnormal immune cell activation has been implicated in disease pathogenesis, including systemic lupus erythematosus (SLE), rheumatoid arthritis (RA), Sjogren's syndrome, and Graves' disease (Karnell et al., 2019b). The broad effects of CD40-CD40L interaction on multiple immune and stromal cell types highlight the importance of this pathway in different aspects of an immune response (Elgueta et al., 2009). As a result, regulating this pathway in disease offers great promise in treating a variety of autoimmune diseases.

CD40 is a member of the TNF-receptor superfamily and is known primarily as a costimulatory receptor that regulates the activation of immune cells as well as non-hematopoietic stromal cells, such as myofibroblasts, fibroblasts, epithelial, and endothelial cells (van Kooten and Banchereau, 1997, 2000). The physiological ligand for CD40- CD40L (also called CD154)—is a constitutively-expressed cellsurface molecule on activated T cells, B cells, and platelets and is induced under inflammatory conditions on monocytes and natural killer (NK) cells (Aloui et al., 2014; Carbone et al., 1997; Gauchat et al., 1995; Gauchat et al., 1993; Grewal and Flavell, 1996). Although the short cytoplasmic tail of CD40 has no intrinsic enzymatic activity, members of TNFR-associated factors (TRAF) protein family couple CD40 to intracellular signaling components. Engagement of CD40 with CD40L leads to recruitment of TRAF proteins to the multimerized cytoplasmic domain of $\mathrm{CD} 40$, which activates the nuclear factor $\kappa \mathrm{B}(\mathrm{NF}$ $\kappa \mathrm{B}$ ), mitogen-activated protein kinases (MAPKs), and phosphoinositide 3-kinase (PI3K) downstream 
pathways to regulate the functions of CD40-mediated responses in a cell-type- and microenvironmentdependent manner (Bishop et al., 2007).

Expression or activity of CD40 and/or CD40L has been shown to be dysregulated in SLE, RA, Psoriatic Arthritis, and Graves' disease, and these changes have been correlated with poor clinical outcomes (Boumpas et al., 2003; Huber et al., 2012; Kanmaz et al., 2004; Karnell et al., 2019b; Voynova et al., 2018). Blockade of this pathway by anti-CD40 and anti-CD40L antibodies has demonstrated therapeutic benefit in SLE, ITP, Graves' Disease, and RA in clinical trials (Boumpas et al., 2003; Kahaly et al., 2020; Kanmaz et al., 2004; Karnell et al., 2019a; Patel et al., 2008). Together, these results support a role for the CD40 pathway in autoimmune disease and suggest that aberrant CD40 signaling can contribute to the initiation or maintenance of pathogenic autoimmune responses (Karnell et al., 2019b).

Initial attempts utilizing non-silenced IgG1 antibodies to target CD40L resulted in thromboembolic events (Boumpas et al., 2003; Law and Grewal, 2009) which were linked to Fc-mediated platelet activation and aggregation (Robles-Carrillo et al., 2010). Recent approaches for blockade of CD40-CD40L interactions to mitigate this safety concern have involved either optimized antibody scaffolds which cannot induce Fc-mediated signaling (if targeting CD40L) or otherwise targeting CD40 itself (Kahaly et al., 2020; Karnell et al., 2019a; Schwabe et al., 2018).

Previous studies with 2C10R4, a chimeric mouse-rhesus monoclonal anti-CD40 antibody, have shown significant prolonged islet allograft survival and xenograft tolerance in nonhuman primate models of transplantation (Lowe et al., 2012; Mohiuddin et al., 2016). KPL-404 is a humanized version of 2C10R4 with an IgG4 constant region chosen to avoid effector function. KPL-404 was previously demonstrated to inhibit CD40/CD40L-mediated B cell activation in peripheral blood mononuclear cells (PBMCs) from healthy volunteers and patients with SLE or Sjogren's syndrome (Marken et al., 2021). Here we report further in vitro and in vivo preclinical characterization of KPL-404 as a highly potent and efficacious CD40 antagonist with a favorable pharmacokinetic profile but without effector function (i.e., without activation or depletion of B cells nor platelet activation). 


\section{MATERIALS AND METHODS}

KPL-404 is a humanized antibody generated by grafting of CDRs from murine 2C10 antibody (Lowe et al., 2012) into human germline antibody sequences and expressed as a stabilized S228P-IgG4 antibody. Of note, $2 \mathrm{C} 10 \mathrm{R} 4$ widely used in primate studies is a chimeric version of $2 \mathrm{C} 10$ comprised by the mouse variable domain of $2 \mathrm{C} 10$ and a rhesus IgG4 constant region. The in vitro and in vivo studies and results were collected and analyzed according to a prespecified plan (including replicate and animal numbers) to define the preclinical profile of KPL-404.

\section{Surface plasmon resonance analysis}

The binding kinetics of KPL-404 to both recombinant human (10774-H08H, Sino Biologicals, Pennsylvania, USA) and cynomolgus monkey (CD0-C52H6, ACROBio, Delaware, USA) CD40 molecules were analyzed using the IBIS MX96 following direct immobilization of KPL-404 onto a Xantec SPMX CMD50 sensor chip. Immobilization was performed via amine coupling — at both 10 $\mu \mathrm{g} / \mathrm{mL}$ and $20 \mu \mathrm{g} / \mathrm{mL}$ loading densities - in $10 \mathrm{mM}$ acetate, $\mathrm{pH} 4.5$, using the CFM printer. Analytes (huCD40 and cyCD40) were run in a kinetics series of $500 \mathrm{nM}, 250 \mathrm{nM}, 125 \mathrm{nM}$ and $62.5 \mathrm{nM}$. The data were analyzed using a 1:1 bimolecular interaction model to calculate association and dissociation rates. The $\mathrm{K}_{\mathrm{D}}$ [equilibrium dissociation constant $=\mathrm{K}_{\mathrm{off}}($ dissociation rate $) / \mathrm{K}_{\mathrm{on}}($ association rate $)$ ] was calculated using Carterra's KIT analysis software.

\section{Cell-based assay}

Human Embryonic Kidney (HEK)-blue CD40L cells (hkb-cd40, Invivogen, California, USA) passaged in DMEM media were plated at 50,000 cells/well in HEK Blue Detection Media (hb-det3, Invivogen, California, USA). The cells were pretreated with isotype antibody control or KPL-404 at the indicated concentration and incubated at $37^{\circ} \mathrm{C} 5 \% \mathrm{CO}_{2}$ for 30 minutes prior to addition of recombinant $\mathrm{CD} 40 \mathrm{~L}$ (BioLegend, California, USA) at $100 \mathrm{ng} / \mathrm{ml}$. OD at $655 \mathrm{~nm}$ was measured after 24 hours for reporter activity. Data were analyzed using GraphPad Prism 4-PL log fit \pm SEM. 


\section{Antibody Dependent Cellular Cytotoxicity (ADCC) and Complement Dependent Cytotoxicity (CDC)}

Human B lymphoblastoid Raji cells were stained with KPL-404 or isotype control antibody at indicated concentrations followed by staining with secondary Alexa Fluor ${ }^{\circledR}$ 488-conjugated anti-human IgG antibody at a dilution of 1:1000 (A11013, Invitrogen, California, USA). The mean fluorescence intensity (MFI) was measured by NovoCyte to evaluate CD40 expression on cells.

For ADCC experiments, peripheral blood mononuclear cells (PBMCs) were isolated from healthy human donor blood to serve as effector cells and were plated with target Raji cells at E:T ratio of 50:1 in the presence of indicated concentrations of KPL-404, anti-CD20 (A2009, Selleck, Washington, USA), or isotype antibodies. Target Raji cells were labeled with calcein-AM (Invitrogen) for detection of cell lysis prior to experimental set up. Following incubation for 4 hours at $37^{\circ} \mathrm{C} 5 \% \mathrm{CO}_{2}$, supernatants from the experiment were analyzed for fluorescence at $485 \mathrm{~nm}$ for detection of cell lysis.

For CDC experiments, human Complement (A113, Quidel) was plated with calcein-AM labeled target Raji cells in the presence of indicated concentrations of KPL-404, anti-CD20, or isotype antibodies. Following incubation for 4 hours at $37^{\circ} \mathrm{C} 5 \% \mathrm{CO}_{2}$, supernatants from the experiment were analyzed for fluorescence at $485 \mathrm{~nm}$ for detection of cell lysis.

The percentage of cell lysis for each group was calculated as follows: $\%$ cell lysis $=100 \mathrm{x}$ (fluorescence of antibody-treated groups $\div$ fluorescence of cells lysed by $2 \%$ Triton X-100 (maximum lysis)).

\section{Method for Detecting Concentration of KPL-404 in Cynomolgus Monkey Serum}

An Enzyme-Linked Immunosorbent Assay (ELISA) method was developed, validated, and used to determine the concentration of KPL-404 in cynomolgus monkey samples. Briefly, Nunc Maxisorp plates (44-2404-21, ThermoFisher Scientific, Grand Island, NY) were coated with a capture protein solution containing human CD40 recombinant protein (10774-H08H, Sino Biologicals, Pennsylvania, USA). After overnight incubation, the plates were washed and blocked with a SuperBlock blocking solution (37515, 
ThermoFisher Scientific, Grand Island, NY). Samples were diluted by minimum required dilution (MRD) of 25-fold with 1X PBS dilution buffer (10010023, ThermoFisher Scientific, Grand Island, NY). In some cases, samples that needed further dilution beyond MRD were diluted with $4 \%$ pooled blank serum (Altasciences Preclinical Seattle LLC, Seattle WA). Following the blocking incubation, the plates were washed, and the diluted samples were added to the plates. Following a 1-hour sample incubation step, the plates were washed, and an anti-human IgG (H+L) HRP solution (NB7489, Novusbio, Centennial, CO) was added. After incubation with the detection antibody for 1 hour, the plates were washed, and a TMB substrate (34029, ThermoFisher Scientific, Grand Island, NY) was added to the plates. After incubation for 10 minutes, a sulfuric acid stopping solution (8310-32, Ricca Chemical, Arlington, TX) was added, and the plates were read using a BioTek Synergy 2 spectrophotometric plate reader (Biotek, Winooski, VT) at an absorbance at $450 \mathrm{~nm}$. A calibration curve was constructed by applying the absorbance at 450 nm of calibration standard samples to a fit type: 4-parameter logistic curve with a $1 / Y$ weighting factor using BioTek's Gen5 Data Analysis Software (Biotek, Winooski, VT). The quantification range of the assay was $80 \mathrm{ng} / \mathrm{mL}$ to $640 \mathrm{ng} / \mathrm{mL}$. The concentrations of KPL-404 in study samples were calculated using the calibration curve generated from the calibration standard samples on the same plate as the study samples.

\section{Method for Detection of Anti-KPL-404 Antibodies (ADA) in Cynomolgus Monkey Serum}

A validated Electrochemiluminescent Immunoassay (ECLIA) method with acid dissociation was used for detection of anti-KPL-404 antibodies in cynomolgus monkey serum. Briefly, samples were incubated with biotinylated KPL-404 (Altasciences Preclinical Seattle LLC, Seattle WA) overnight. On the second day, a separate streptavidin high binding capacity extraction plate (ThermoFisher Scientific, Grand Island, NY) was blocked, and a mixture of samples and biotinylated KPL-404 prepared on Day 1 was transferred to the extraction plate and incubated for one hour. Following incubation, acetic acid (VWR International, Radnor PA) was added to the plate, and samples were transferred to a polypropylene plate prefilled with Tris buffer (VWR International, Radnor PA) for the screening assay or KPL-404-spiked Tris buffer for 
confirmatory assay and incubated for at least 30 minutes (extracted samples). A Streptavidin Gold MSD assay plate (Meso Scale Diagnoastics, Rockville, Maryland) was blocked for 1 to 4 hours followed by addition of biotinylated KPL-404 to the plate and incubated 1 hour. Following this incubation, the assay plate was washed, and the extracted samples were transferred from the polypropylene plate to the MSD assay plate and incubated on a plate shaker for 1 hour. The plate was washed, and ruthenylated KPL-404 (Altasciences Preclinical Seattle LLC, Seattle WA) was added, covered with aluminum foil, and incubated for 1 hour. After incubation, the MSD assay plate was washed, and MSD read buffer (Meso Scale Diagnostics, Rockville, Maryland) was added to the plate for reading on the MSD SECTOR Imager (Meso Scale Diagnostics, Rockville, Maryland). Mean luminescence intensity and \%CV of the duplicate sample luminescence was calculated using MSD Discovery Workbench (Meso Scale Diagnostics, Rockville, Maryland), and the statistical cut-points established during validation were applied to determine samples with positive and negative responses.

\section{In vivo studies}

Pharmacokinetic/pharmacodynamic (PK/PD) studies performed at Altasciences (formerly SNBL; Washington, USA) utilized biologics-treatment-naive cynomolgus monkeys (Macaca fascicularis) 2.5-5 years old $(2.5 \pm 3.5 \mathrm{~kg}, 6$ males and 36 females). Animals were supplied by Orient BioResource Center and originated from Cambodia. Animals were maintained at the Testing Facility (Everett, WA facility) as stock prior to study assignment and were screened for health by veterinary staff prior to use on study. PMI's LabDiet ${ }^{\circledR}$ Fiber-Plus ${ }^{\circledR}$ Monkey Diet 5049 biscuits and fresh water were provided ad libitum. All animal studies complied with the National Institutes of Health Guide for the Care and Use of Laboratory Animals (NIH Publications No. 8023, revised 1978), the 'Animal Research: Reporting of In Vivo Experiments' guidelines and applicable animal welfare acts. Altasciences Laboratories is fully accredited by the Association for Assessment and Accreditation of Laboratory Animal Care and studies were approved by the local Institutional Animal Care and Use Committee. Animal handling, care, drug treatments, and blood sampling were performed according to an authorized study protocol. 
Cynomolgus monkeys ( $n=7$ animals/group) received one or two doses (separated by 4 weeks) of KPL$404(1,5$, or $10 \mathrm{mg} / \mathrm{kg})$ intravenously over the study duration of 56 days. The effect of KPL-404 on antigen priming/re-challenge model was also tested by administration of $\mathrm{T}$ cell-dependent antigens Keyhole limpet hemocyanin (KLH) (Imject Mariculture KLH, ThermoFisher Scientific, Massachusetts, USA) and tetanus toxoid (TT) (Tenivac, Sanofi-Pasteur, Pennsylvania, USA) in these cynomolgus monkeys (Figure 1). Placebo or KPL-404 was administered intravenously at 1,5 , or $10 \mathrm{mg} / \mathrm{kg}$ on day 0 followed by KLH (1 mg/animal) + TT (5 Lf/animal) priming intramuscularly at 3 hours post-KPL404/placebo dose on day 0. Placebo- or KPL-404- (1 and $5 \mathrm{mg} / \mathrm{kg}$ ) dosed animals received a second dose and a second KLH (0.5 mg/animal) + TT antigen (5 Lf/animal) challenge on day 28 . In separate cohorts, animals were primed with KLH on day 0 (without KPL-404 or placebo) and re-challenged with KLH on day 28 at 3 hours post placebo or KPL-404 $5 \mathrm{mg} / \mathrm{kg}$ administration. Serum samples collected as scheduled were analyzed for KPL-404 serum pharmacokinetics (PK), KPL-404 anti-drug antibodies (ADA), and anti-KLH/TT IgM and IgG responses (ELISA). KPL-404 CD40 receptor occupancy (RO) in blood was assessed by flow cytometry.

Blood samples collected for RO were split into two tubes for analysis by flow cytometry. Cells were stained with CD20 PerCP-Cy5.5 (BD Biosciences, New Jersey, USA) and either CD40 specific C40/1605 AF647 or G28.5 AF647 (Novus Biologicals, Missouri, USA). Data were analyzed by gating on lymphocytes first then on CD20+ B cells to examine \% cells positive for CD40. C40/1605 competes with KPL-404 for binding to CD40, and positive staining is observed only in the absence of KPL-404, indicating \% cells with unoccupied (free) CD40. G28.5 binds CD40 in the absence or presence of CD40, indicating \% all cells expressing CD40. KPL-404-occupied CD40 (RO) was calculated by percentage of $\% \mathrm{C} 40 / 1605$ positive cells over \%G28.5 positive cells.

Monkey anti-KLH and anti-TT IgM and IgG serum concentrations were determined using commercial ELISA kits manufactured by Life Diagnostics, Pennsylvania, USA (KLHM-3 and KLHG-3-INT), Alpha Diagnostic International, Texas, USA (930-415-TKM and 930-410-TKG). Monkey serum samples were 
thawed and diluted in sample diluent immediately prior to testing. A minimum dilution of 1:500 was used for testing in the anti-KLH IgM and the anti-KLH IgG ELISA. A minimum dilution of 1:1000 for the anti-TT IgM ELISA, and a minimum dilution of 1:200 was used for the anti-TT IgG ELISA. Standard curves were generated in SoftMax®Pro by plotting the mean A450 values (y-axis) versus concentration (x-axis) and fitting the curve using the 4-parameter logistic (anti-KLH) and point-to-point (anti-TT) function. Concentrations of anti-KLH and anti-TT $\operatorname{IgM}$ and $\operatorname{IgG}$ in test samples were obtained by multiplying the mean interpolated value from duplicate wells by the sample dilution factor. 


\section{$\underline{\text { RESULTS }}$}

\section{KPL-404 binds CD40 and inhibits CD40 downstream signaling in vitro}

Binding kinetics of KPL-404 for human and cynomolgus monkey CD40 molecules, as determined by surface plasmon resonance (SPR) studies, were comparable for human and cynomolgus monkey CD40 (association rate constant $\left[\mathrm{K}_{\mathrm{on}}\right]$ of $2.5 \times 10^{4} \mathrm{M}^{-1} \mathrm{~s}^{-1}$ and a disassociation rate constant $\left[\mathrm{K}_{\mathrm{off}}\right]$ of $1.8 \times 10^{-4} \mathrm{~s}^{-1}$ for human CD40, and a $\mathrm{K}_{\text {on }}$ of $5 \times 10^{4} \mathrm{M}^{-1} \mathrm{~s}^{-1}$ and a $\mathrm{K}_{\text {off }}$ of $1.48 \times 10^{-4} \mathrm{~s}^{-1}$ for cynomolgus monkey CD40). KPL-404 bound both human and cynomolgus monkey CD40 with comparable affinity with $\mathrm{K}_{\mathrm{D}}$ of $7.2 \mathrm{nM}$ and $2.9 \mathrm{nM}$, respectively (Figure 2A). Binding of KPL-404 to recombinant human CD40 was further confirmed in an ELISA format. KPL-404 bound to immobilized CD40 (CD40 with His tag or CD40 with Fc tag) was detected using anti-human IgG4, and a dose-response curve showed that the $\mathrm{EC}_{50}$ of KPL-404 binding to plate-bound recombinant human CD40 was $14 \mathrm{ng} / \mathrm{mL}$ (Figure 2B).

KPL-404 inhibited signaling downstream of CD40 triggered by binding of CD40L in an engineered cell line, HEK-blue CD40L cells, which express human CD40 and an NF- $\mathrm{BB}$-inducible reporter construct. Recombinant soluble CD40L binding to cell-surface CD40 activates downstream signaling and transcription factor $\mathrm{NF}-\mathrm{\kappa B}$, which results in production of secreted embryonic alkaline phosphatase (SEAP). The $\mathrm{IC}_{50}$ for KPL-404 inhibition of CD40-CD40L-induced downstream reporter activity was $3.5 \mathrm{nM}$ at $100 \mathrm{ng} / \mathrm{mL}$ CD40L stimulation, while an IgG4 isotype control had no inhibitory effect on CD40-CD40L-induced NF- $\mathrm{B}$ activation (Figure 2C). Therefore, KPL-404 binds CD40 and inhibits CD40L-mediated downstream signaling.

\section{KPL-404 does not induce lysis of CD40-expressing Raji cells in vitro}

KPL-404 was engineered to be a CD40 antagonist antibody that does not deplete B-cells, given the broad expression of CD40 on multiple cell-types; this non-depleting attribute was confirmed in in vitro ADCC and CDC studies. The human B cell line, Raji, which expresses high levels of CD40, bound KPL-404 in a concentration-dependent manner as determined by flow cytometry (Figure 3A). KPL-404 
was incubated with CD40-expressing Raji cells as the target cells and human PBMCs as effector cells in testing ADCC or human complement as effector molecules in testing $\mathrm{CDC}$, and target cell lysis was evaluated. Anti-CD20 IgG1 antibody (rituximab), the positive control for the assay, induced strong $\mathrm{ADCC}$ and $\mathrm{CDC}$ effects on $\mathrm{CD} 20+$ Raji cells with the $\mathrm{EC}_{50}$ at $0.0365 \mathrm{nM}$ and $1.5 \mathrm{nM}$, respectively (Figure 3B-3C). As expected, KPL-404 did not induce target-cell lysis via ADCC or CDC at any concentration tested.

\section{KPL-404 engages CD40 target and shows favorable pharmacokinetics in vivo}

Considering the comparable affinity of KPL-404 for human and monkey CD40 in vitro, the cynomolgus monkey was a suitable species to perform in vivo studies to evaluate KPL-404 PK-PD relationships and safety. The 8-week in vivo study design is outlined in Figure 1. KPL-404, given as a single $10 \mathrm{mg} / \mathrm{kg}$ intravenous dose or two 1 or $5 \mathrm{mg} / \mathrm{kg}$ doses (separated by 4 weeks), was well-tolerated.

In order to determine peripheral drug exposure levels, KPL-404 concentrations in serum following IV administration were examined on a weekly basis for 8 weeks. The mean for maximum serum KPL-404 concentration $\left(\mathrm{C}_{\max }\right)$ was $26 \mu \mathrm{g} / \mathrm{ml}, 138 \mu \mathrm{g} / \mathrm{ml}$, and $273 \mu \mathrm{g} / \mathrm{ml}$ after first dose for 1,5 , and $10 \mathrm{mg} / \mathrm{kg}$ groups respectively. Exposure levels remained $>1 \mu \mathrm{g} / \mathrm{ml}$ for 1,4 , and 6 weeks for 1,5 , and $10 \mathrm{mg} / \mathrm{kg}$ IV group, respectively, after a single dose (Figure $4 \mathrm{~A}$, Table 1). Comparisons of $\mathrm{C}_{\max }$ and AUC across dose groups following repeat dose administration were limited by the pronounced impact of ADAs in all animals in the lower dose group $(1 \mathrm{mg} / \mathrm{kg})$ and some animals in the middle dose group $(5 \mathrm{mg} / \mathrm{kg})$. The nonlinear PK profiles and concentration dependent clearance rates indicate target-mediated drug disposition (TMDD)(Huber et al., 2012; Karnell et al., 2019b).

KPL-404 binding to target CD40, i.e., receptor occupancy (RO), on B cells in whole blood was measured by flow cytometry using a pair of antibodies recognizing either total CD40 or unoccupied CD40 (non-KPL-404-bound) on a weekly basis for 8 weeks. Full (>95\%) RO by KPL-404 (at KPL-404 serum concentrations of $>2 \mu \mathrm{g} / \mathrm{mL}$ ), confirming KPL-404 binding to $>95 \%$ of B cell-expressed CD40 in 
peripheral blood, was observed in all animals up to 7 days, 14 days, and 28 days in 1,5 , and $10 \mathrm{mg} / \mathrm{kg}$ dose groups respectively, demonstrating that the magnitude of target engagement by KPL-404 correlates with serum concentration profiles (Figure 4B, Supplementary Figure 1).

Presence of ADAs against KPL-404 was evaluated in serum at pre-dose, 4 weeks, and 8 weeks postKPL-404 administration. At 4 weeks post-dose, the first timepoint tested, anti-drug antibodies (ADAs) were detectable in all 7 animals receiving the lowest dose level $(1 \mathrm{mg} / \mathrm{kg})$. While animals receiving higher doses appeared less likely to have detectable ADAs at this timepoint (4 of 7 recipients of $5 \mathrm{mg} / \mathrm{kg}$ and 3 of 7 recipients of $10 \mathrm{mg} / \mathrm{kg}$ ), it should be noted that high drug concentrations in the plasma confounded the assay by competing with ADA for binding, rendering the results in the high dose group inconclusive. Corresponding to decline in KPL-404 serum levels over time, 20 of 21 animals receiving KPL-404 tested positive for ADAs by 8 weeks post-dose, the end of the study (Figure 4B).

In this in vivo study, after a single IV administration of KPL-404, there were no KPL-404-related clinical observations, changes in body weight or hematology, including thrombocytopenia, nor adverse injection site changes in the cynomolgus monkeys based on gross inspection and microscopic examination of skin punch biopsies. Consistent with in vitro observations, KPL-404 did not induce depletion of peripheral CD20+ B cells (Figure 5). The absence of observable safety findings suggests a good safety profile for KPL-404 at indicated exposure levels.

\section{KPL-404 suppresses antigen-specific antibody responses in vivo}

Since the CD40-CD40L pathway is required for antigen-primed B cells to become activated and to form GCs which generate class-switched, affinity matured IgG antibodies in response to $\mathrm{T}$ celldependent antigens, an antigen challenge model was used to evaluate the pharmacodynamic efficacy of KPL-404 to block T-cell-dependent antibody response.

Suppression of T-cell-dependent primary anti-KLH and anti-TT IgM responses was observed through 28 days in both KPL-404 5 and $10 \mathrm{mg} / \mathrm{kg}$-dosed animals compared to control animals that 
received placebo (Figure 6A, 6B). Primary anti-KLH and -TT IgG levels were also suppressed in KPL4045 and $10 \mathrm{mg} / \mathrm{kg}$-dosed animals compared to control animals (Figure 6C, 6D).

In evaluation of the secondary immune response, the second administration of KPL-404 at the 5 $\mathrm{mg} / \mathrm{kg}$ dose level suppressed secondary anti-KLH and anti-TT IgG compared to control placebo group upon antigen rechallenge, after having excluded samples from animals that tested ADA-positive at the time of antigen rechallenge to compensate for observed rapid loss of KPL-404 serum levels and CD40 target engagement (Figure 6C, 6D).

The efficacy of KPL-404 in blocking the recall (secondary) antibody response in the absence of prior blockade of primary antibody responses was evaluated in separate cohorts in the PK-PD study. The PK and RO profiles of the animals in this cohort (Figure 7A, 7B) were similar to the cohort dosed with KPL-404 $5 \mathrm{mg} / \mathrm{kg}$ on Day 0 (Figure 4A, 4B). In the case of antigen priming in the absence of CD40 blockade, KPL-404 $5 \mathrm{mg} / \mathrm{kg}$ suppressed secondary anti-KLH IgG recall response by $41 \%$ compared to control animals at day 42, which is the peak of response (Figure 7C). 


\section{DISCUSSION}

The CD40 antagonist antibody, KPL-404, blocks activation of the CD40/CD40L pathway, which has been implicated in multiple autoimmune diseases including SLE, RA, Sjogren's Syndrome, and Graves' Disease. KPL-404 has been shown to block in vitro CD40-CD40L-mediated activation of B cells from the blood of healthy as well as SLE and Sjogren's Syndrome donors (Marken et al., 2021). Here, we have further characterized the binding and functional properties of KPL-404 in vitro and demonstrated its preclinical in vivo pharmacokinetic and pharmacodynamic profile in support of its development as a potential therapeutic in autoimmune diseases.

In vitro experiments showed that KPL-404 bound recombinant CD40 as determined by SPR and cell-surface-expressed CD40 as determined by flow cytometry. This was consistent with the previous in vitro study by Marken et al. which showed binding of KPL-404 to CD40 expressed on B cells and monocytes from peripheral blood. It should be noted that KPL-404 bound to recombinant CD40 molecules with higher affinity $\left(\mathrm{K}_{\mathrm{D}} 7 \mathrm{nM}\right)$ than the natural affinity of CD40L for CD40, which has been reported to be 10-30 $\mathrm{nM}\left(\mathrm{K}_{\mathrm{D}}\right)$ by SPR. Epitope mapping studies indicated KPL-404 binds CD40 at amino acids $\mathrm{Glu}^{28}, \mathrm{Asp}^{69}, \mathrm{Arg}^{73}$, and $\mathrm{Glu}^{74}$ on adjacent loops of the extracellular domain (Michaels et al., 2019). This epitope is $100 \%$ conserved between human and cynomolgus monkey CD40, and several amino acids in this region are involved in CD40-CD40L interaction as shown from crystal structure, suggesting possible steric mechanism of action for KPL-404 inhibition of CD40-CD40L interaction. Indeed KPL404 inhibited CD40L-induced activation of CD40 downstream signaling but did not induce depletion of CD40-expressing cells in vitro, which may be helpful from a safety perspective when targeting CD40 clinically, given the broad expression profile of this receptor on immune and stromal cell types. Absence of in vitro human B cell activation by KPL-404 has also been demonstrated previously (Marken et al., 2021). In vitro studies with human platelets have shown KPL-404 does not induce platelet activation (CD62P expression), aggregation or dense granule release in the presence or absence of soluble CD40L (data not shown). We did not observe thrombocytopenia over the 8-week in vivo study. These 
observations are in line with the expected pharmacological profile of KPL-404 as a true antagonistic antibody targeting the CD40-CD40L pathway and consistent with the previous study using peripheral blood mononuclear cells from healthy donors and SLE and Sjogren's Syndrome patients (Marken et al., 2021).

In vivo PK profiles indicated dose-dependent target mediated drug disposition (TMDD). The terminal half-life of KPL-404 could not be calculated due to these TMDD effects and the impact of crossspecies ADAs on PK profiles. Considering KPL-404 is a humanized antibody being administered to monkeys, development of ADAs against drug product in these animals is not unexpected. While therapeutic CD40 antagonism does block generation of antibodies against neoantigens, including KPL404, ADAs can form over time when the concentration of CD40 antagonist falls below pharmacologically relevant levels needed to suppress antibody formation in lymph nodes, which is expected to be inversely proportional to dose. In this study, samples were not collected for ADA detection until 28 days post-dose. CD40 inhibition, if therapeutic, would be expected to block ADA formation similar to other T-cell dependent antibody response (TDAR) during this timeframe, and inhibition of antibody generation to the neo-antigens TT and KLH were also observed during this time window. At Day 28, ADAs were detected in all animals receiving the lowest dose and less than half of the animals receiving the highest dose, which is consistent with the mechanism of suppression of antibodies by CD40 antagonism. However, it should be noted that high drug concentrations in the plasma confounded the assay by competing with ADA for binding, rendering the results in the high dose group inconclusive. Nearly all animals had ADA detected by 8 weeks post dose. The appearance of ADAs in these animals could account for lower than expected PK after the second dose potentially due to more rapid elimination, thus impacting KPL-404 PK and RO. Indeed KPL-404 serum concentrations were decreased more rapidly, and KPL-404 RO was also lost more rapidly following the second dose in the 1 and $5 \mathrm{mg} / \mathrm{kg}$ dose group potentially due to ADA interference. These preclinical KPL-404 PK and RO profiles, taking into account these TMDD effects at clinically 
relevant doses such as 5 and $10 \mathrm{mg} / \mathrm{kg}$, can contribute to PK modeling for KPL-404 clinical development and determination of dosing regimens for clinical trials.

The observed timing and PK decay curves correlated with loss of full RO across doses, implicating a threshold concentration of $2 \mu \mathrm{g} / \mathrm{ml}$ needed to achieve $\geq 90 \% \mathrm{RO}$ in peripheral blood in cynomolgus monkeys. However, the more physiologically relevant pharmacodynamic effects of KPL-404 would be exerted in tissue rather than peripheral blood such as in inhibition of GC formation and antibody class switching in response to $\mathrm{T}$ cell-dependent antigens in lymph nodes; therefore, the plasma concentrations that correlate with these in-tissue (or site-of-action) PD effects must be determined empirically. KPL-404 effects on anti-KLH and anti-TT IgG antibody responses after neoantigen challenge were measured as PD markers. Suppression of antigen-specific antibody responses, both primary and secondary, by KPL-404 were observed at serum KPL-404 concentrations of >20 $\mu \mathrm{g} / \mathrm{ml}$ which was maintained for 2 and 4 weeks at doses of 5 and $10 \mathrm{mg} / \mathrm{kg}$ respectively (Supplementary Figure 2). This could also be correlated to development of ADAs in some animals in the KPL-404 $5 \mathrm{mg} / \mathrm{kg}$ and $10 \mathrm{mg} / \mathrm{kg}$ group by day 28 as a result of serum levels of $<20 \mu \mathrm{g} / \mathrm{ml} \mathrm{KPL}-404$. This indicates serum levels of KPL-404 of $20 \mu \mathrm{g} / \mathrm{ml}$ and higher are needed to inhibit CD40 in tissues where this pathway is more relevant for GC formation and antibody production. Such a PK-PD relationship is in line with previous observations for another CD40 antagonist antibody CFZ533 (iscalimab) wherein the authors reported 20fold higher peripheral drug levels were needed for suppression of GCs and antigen-specific antibody responses than for full target occupancy in peripheral blood (Ristov et al., 2018). In that study with 4 monkeys, CFZ533 demonstrated suppression of secondary anti-KLH IgG responses correlated with 40 $\mu \mathrm{g} / \mathrm{ml} \mathrm{CFZ533} \mathrm{serum} \mathrm{concentrations} \mathrm{following} \mathrm{intravenous} \mathrm{administration} \mathrm{of} 10 \mathrm{mg} / \mathrm{kg}$ CFZ533. In another small study with 3 animals, CFZ533 showed $>90 \%$ RO for less than 28 days at doses of 16-20 $\mathrm{mg} / \mathrm{kg}$ administered intravenously with variable PK profiles. A transient decrease in total CD40 expression following CFZ533 administration in vivo, potentially due to internalization or shedding, was 
observed. In our study, total CD40 levels on B cells appeared unaffected during the entire duration of KPL-404 exposure (data not shown), but this hypothesis warrants further experimentation.

We have also shown that KPL-404 inhibits secondary (anamnestic) antibody responses to repeat antigen exposure after a normal antigen-specific priming done in absence of CD40 blockade. These data indicate that the antibody recall or memory $\mathrm{B}$ cell responses in response to $\mathrm{T}$ cell-dependent antigens are also dependent on CD40-CD40L, though the extent is unclear since inhibition is incomplete. This also further strengthens rationale for KPL-404 to at least partially block existing autoantibody responses in autoimmune patients, where the autoimmune response would be an ongoing process with secondary/memory antibody responses to repeated autoantigen exposure, and the primary antibody responses were most likely mounted during disease initiation prior to any therapeutic intervention. It should be noted, however, that KPL-404 would not be expected to block responses of long-lived plasma cells which develop from activated B cells and do not require CD40-CD40L interactions to produce antibodies.

Given the broad spectrum of impact of CD40 signaling on the immune response and its potential role in many autoimmune diseases, several molecules targeting the CD40-CD40L pathway have been generated and evaluated in clinical settings. Trials with CD40 or CD40L-targeting investigational therapeutics such as iscalimab (CFZ533), VIB4920, dapirolizumab pegol, and ruplizumab (BG9588) have demonstrated favorable efficacy in improving EULAR Sjogren's Syndrome disease activity index scores in patients with Sjogren's Syndrome, DAS28-CRP in patients with Rheumatoid Arthritis, SLE activation indexes such as SLEDAI in patients with SLE and T3/T4 levels in patients with Graves' Disease, providing external proof of concept for targeting this pathway in autoimmune disease (Boumpas et al., 2003; Chamberlain et al., 2017; Kahaly et al., 2020; Karnell et al., 2019a). In conclusion, our in vitro and in vivo studies characterize KPL-404 as a blocking anti-CD40 antibody that potently inhibits primary and secondary antibody responses at pharmacologically relevant concentrations in TDAR studies and did not 
deplete B-cells by antibody dependent cellular cytotoxicity or apoptosis ("non-depleting"), supporting clinical development of KPL-404 as a potential therapeutic in autoimmune diseases.

\section{ACKNOWLEDGMENTS}

The authors would like to thank Alexandra Joseph for her contributions during manuscript development.

The authors also acknowledge Emily Plummer, PhD, Kiniksa Pharmaceuticals Corp., for medical writing support.

\section{AUTHORSHIP CONTRIBUTIONS}

Participated in research design: Muralidharan, Njenga, Paolini

Conducted experiments: Muralidharan, Njenga, Garron

Performed data analysis: Muralidharan, Njenga, Garron

Wrote the first draft of the manuscript: Muralidharan

Contributed to substantive review/revision of the manuscript: all authors 


\section{REFERENCES}

Aloui, C., Prigent, A., Sut, C., Tariket, S., Hamzeh-Cognasse, H., Pozzetto, B., Richard, Y., Cognasse, F., Laradi, S., and Garraud, O. (2014). The signaling role of CD40 ligand in platelet biology and in platelet component transfusion. Int J Mol Sci 15: 22342-22364.

Bishop, G.A., Moore, C.R., Xie, P., Stunz, L.L., and Kraus, Z.J. (2007). TRAF proteins in CD40 signaling. Adv Exp Med Biol 597: 131-151.

Boumpas, D.T., Furie, R., Manzi, S., Illei, G.G., Wallace, D.J., Balow, J.E., Vaishnaw, A., and Group, B.G.L.N.T. (2003). A short course of BG9588 (anti-CD40 ligand antibody) improves serologic activity and decreases hematuria in patients with proliferative lupus glomerulonephritis. Arthritis Rheum 48: 719727.

Carbone, E., Ruggiero, G., Terrazzano, G., Palomba, C., Manzo, C., Fontana, S., Spits, H., Karre, K., and Zappacosta, S. (1997). A new mechanism of NK cell cytotoxicity activation: the CD40-CD40 ligand interaction. J Exp Med 185: 2053-2060.

Chamberlain, C., Colman, P.J., Ranger, A.M., Burkly, L.C., Johnston, G.I., Otoul, C., Stach, C., Zamacona, M., Dorner, T., Urowitz, M., et al. (2017). Repeated administration of dapirolizumab pegol in a randomised phase I study is well tolerated and accompanied by improvements in several composite measures of systemic lupus erythematosus disease activity and changes in whole blood transcriptomic profiles. Ann Rheum Dis 76: 1837-1844.

Danese, S., Sans, M., and Fiocchi, C. (2004). The CD40/CD40L costimulatory pathway in inflammatory bowel disease. Gut 53: 1035-1043.

Elgueta, R., Benson, M.J., de Vries, V.C., Wasiuk, A., Guo, Y., and Noelle, R.J. (2009). Molecular mechanism and function of CD40/CD40L engagement in the immune system. Immunol Rev 229: 152172.

Gauchat, J.F., Henchoz, S., Fattah, D., Mazzei, G., Aubry, J.P., Jomotte, T., Dash, L., Page, K., Solari, R., Aldebert, D., et al. (1995). CD40 ligand is functionally expressed on human eosinophils. Eur J Immunol 25: 863-865.

Gauchat, J.F., Henchoz, S., Mazzei, G., Aubry, J.P., Brunner, T., Blasey, H., Life, P., Talabot, D., FloresRomo, L., Thompson, J., et al. (1993). Induction of human IgE synthesis in B cells by mast cells and basophils. Nature 365: 340-343.

Grewal, I.S., and Flavell, R.A. (1996). The role of CD40 ligand in costimulation and T-cell activation. Immunol Rev 153: 85-106.

Hernandez, M.G., Shen, L., and Rock, K.L. (2007). CD40-CD40 ligand interaction between dendritic cells and CD8+ T cells is needed to stimulate maximal T cell responses in the absence of CD4+ T cell help. $J$ Immunol 178: 2844-2852. 
Huber, A.K., Finkelman, F.D., Li, C.W., Concepcion, E., Smith, E., Jacobson, E., Latif, R., Keddache, M., Zhang, W., and Tomer, Y. (2012). Genetically driven target tissue overexpression of CD40: a novel mechanism in autoimmune disease. J Immunol 189: 3043-3053.

Inwald, D.P., McDowall, A., Peters, M.J., Callard, R.E., and Klein, N.J. (2003). CD40 is constitutively expressed on platelets and provides a novel mechanism for platelet activation. Circ Res 92: 1041-1048. Kahaly, G.J., Stan, M.N., Frommer, L., Gergely, P., Colin, L., Amer, A., Schuhmann, I., Espie, P., Rush, J.S., Basson, C., et al. (2020). A Novel Anti-CD40 Monoclonal Antibody, Iscalimab, for Control of Graves Hyperthyroidism-A Proof-of-Concept Trial. J Clin Endocrinol Metab 105.

Kanmaz, T., Fechner, J.J., Jr., Torrealba, J., Kim, H.T., Dong, Y., Oberley, T.D., Schultz, J.M., Bloom, D.D., Katayama, M., Dar, W., et al. (2004). Monotherapy with the novel human anti-CD154 monoclonal antibody ABI793 in rhesus monkey renal transplantation model. Transplantation 77: 914-920.

Karnell, J.L., Albulescu, M., Drabic, S., Wang, L., Moate, R., Baca, M., Oganesyan, V., Gunsior, M., Thisted, T., Yan, L., et al. (2019a). A CD40L-targeting protein reduces autoantibodies and improves disease activity in patients with autoimmunity. Sci Transl Med 11.

Karnell, J.L., Rieder, S.A., Ettinger, R., and Kolbeck, R. (2019b). Targeting the CD40-CD40L pathway in autoimmune diseases: Humoral immunity and beyond. Adv Drug Deliv Rev 141: 92-103.

Law, C.L., and Grewal, I.S. (2009). Therapeutic interventions targeting CD40L (CD154) and CD40: the opportunities and challenges. Adv Exp Med Biol 647: 8-36.

Lowe, M., Badell, I.R., Thompson, P., Martin, B., Leopardi, F., Strobert, E., Price, A.A., Abdulkerim, H.S., Wang, R., Iwakoshi, N.N., et al. (2012). A novel monoclonal antibody to CD40 prolongs islet allograft survival. Am J Transplant 12: 2079-2087.

Marken, J., Muralidharan, S., and Giltiay, N.V. (2021). Anti-CD40 antibody KPL-404 inhibits T cellmediated activation of B cells from healthy donors and autoimmune patients. Arthritis Res Ther 23: 5 .

Michaels, A.J., Stoppato, M., Flores, W.J., Reimann, K.A., and Engelman, K.D. (2019). Anti-CD40 antibody $2 \mathrm{C} 10$ binds to a conformational epitope at the CD40-CD154 interface that is conserved among primate species. Am J Transplant.

Mohiuddin, M.M., Singh, A.K., Corcoran, P.C., Thomas lii, M.L., Clark, T., Lewis, B.G., Hoyt, R.F., Eckhaus, M., Pierson lii, R.N., Belli, A.J., et al. (2016). Chimeric 2C10R4 anti-CD40 antibody therapy is critical for long-term survival of GTKO.hCD46.hTBM pig-to-primate cardiac xenograft. Nat Commun 7: 11138.

Patel, V.L., Schwartz, J., and Bussel, J.B. (2008). The effect of anti-CD40 ligand in immune thrombocytopenic purpura. Br J Haematol 141: 545-548.

Phipps, R.P. (2008). CD40: Lord of the endothelial cell. Blood 112: 3531-3532.

Ristov, J., Espie, P., Ulrich, P., Sickert, D., Flandre, T., Dimitrova, M., Muller-Ristig, D., Weider, D., Robert, G., Schmutz, P., et al. (2018). Characterization of the in vitro and in vivo properties of CFZ533, a blocking and non-depleting anti-CD40 monoclonal antibody. Am J Transplant 18: 2895-2904. 
Robles-Carrillo, L., Meyer, T., Hatfield, M., Desai, H., Davila, M., Langer, F., Amaya, M., Garber, E., Francis, J.L., Hsu, Y.M., et al. (2010). Anti-CD40L immune complexes potently activate platelets in vitro and cause thrombosis in FCGR2A transgenic mice. J Immunol 185: 1577-1583.

Schwabe, C., Rosenstock, B., Doan, T., Hamilton, P., Dunbar, P.R., Eleftheraki, A.G., Joseph, D., Hilbert, J., Schoelch, C., Padula, S.J., et al. (2018). Safety, Pharmacokinetics, and Pharmacodynamics of Multiple Rising Doses of BI 655064, an Antagonistic Anti-CD40 Antibody, in Healthy Subjects: A Potential Novel Treatment for Autoimmune Diseases. J Clin Pharmacol 58: 1566-1577.

van Kooten, C., and Banchereau, J. (1997). Immune regulation by CD40-CD40-L interactions. Front Biosci 2: d1-11.

van Kooten, C., and Banchereau, J. (2000). CD40-CD40 ligand. J Leukoc Biol 67: 2-17.

Voynova, E., Mahmoud, T., Woods, L.T., Weisman, G.A., Ettinger, R., and Braley-Mullen, H. (2018). Requirement for CD40/CD40L Interactions for Development of Autoimmunity Differs Depending on Specific Checkpoint and Costimulatory Pathways. Immunohorizons 2: 54-66. 


\section{FUNDING STATEMENT}

The study was sponsored by Kiniksa Pharmaceuticals, Ltd (Bermuda).

\section{FINANCIAL DISCLOSURE}

${ }^{1}$ Sujatha Muralidharan discloses employment by Kiniksa Pharmaceuticals during the study. ${ }^{2}$ Moses Njenga is an employee and stockholder of Kiniksa Pharmaceuticals Corp. ${ }^{3}$ Tracy Garron is an employee and stockholder of Kiniksa Pharmaceuticals Corp. ${ }^{4}$ Kent Bondensgaard is an employee and stockholder of Kiniksa Pharmaceuticals Corp. ${ }^{5}$ John F. Paolini is an employee and stockholder of Kiniksa Pharmaceuticals Corp and is an inventor on patent applications related to mavrilimumab.

These data were previously presented: Muralidhara S, Classon BJ, Crowder K, et al. KPL-404, a CD40 antagonist, blocked antigen-specific antibody responses in an in vivo NHP model and demonstrated strong PK/PD correlation. 2019. Poster presented at Keystone Symposia: Antibodies as Drugs: New Horizons in the Therapeutic Use of Engineered Antibodies. 


\section{FIGURE LEGEND}

\section{Figure 1: Study design for in vivo TDAR model in cynomolgus monkeys}

Placebo or KPL-404 was dosed at indicated dose levels intravenously (i.v.), and KLH and TT antigens administered intramuscularly (i.m.) with 7 animals/group over a 56 day period. On days of administration of both test article and antigens, placebo/KPL-404 was administered first and then KLH/TT antigens were administered 3 hours later. Arrows indicate timepoints for collection of serum/blood samples for PK (purple), RO (green), Ag-specific antibody titers (blue) and ADA (red).

\section{Figure 2: KPL-404 binds CD40 and inhibits CD40 downstream signaling in vitro}

(A) Kinetics of KPL-404 binding to human and cynomolgus monkey CD40 were determined by surface plasmon resonance analysis, and $\mathrm{K}_{\mathrm{D}}$ was determined. Sensorgram plots are raw kinetics data plots for binding at KPL-404 $20 \mu \mathrm{g} / \mathrm{ml}$ surface binding density. (B) ELISA plots of KPL-404 binding to immobilized CD40-His or CD40-Fc at different concentrations were used to determine KPL-404 $\mathrm{EC}_{50}$. (C) Cell-based assay with HEK-Blue CD40L cells pre-incubated with KPL-404 or isotype control antibodies at different concentrations showed inhibition of CD40L stimulated reporter activity which was used to determine KPL-404 $\mathrm{IC}_{50}$.

Figure 3: KPL-404 does not induce lysis of CD40-expressing Raji cells in vitro

(A) CD40 expression on Raji cells was detected by KPL-404 staining for flow cytometry and expressed as mean fluorescent intensity (MFI) or \% cells positive. (B) \%cell lysis induced by ADCC was calculated at different concentrations of KPL-404 or control antibodies. (C) \%cell lysis induced by CDC was calculated at different concentrations of KPL-404 or control antibodies. 
Figure 4: KPL-404 engages CD40 target and shows favorable pharmacokinetics in vivo

(A) KPL-404 serum concentrations in cynomolgus monkeys from in vivo TDAR model were measured over the 56 day study duration. (B) KPL-404 CD40 receptor occupancy was measured in peripheral blood of cynomolgus monkeys from in vivo TDAR model over the 56 day study duration. Animals that tested ADA positive are indicated by red cross square symbols. These data include cohorts that received KPL404 and KLH/TT administration on day 0 (Placebo, 1, 5 and $10 \mathrm{mg} / \mathrm{kg}$ ) and day 28 (Placebo, 1, $5 \mathrm{mg} / \mathrm{kg}$ ). Black arrows indicate placebo/KPL-404 IV administration.

\section{Figure 5: KPL-404 does not induce depletion of CD20+ B cells}

CD20+ B cell counts in peripheral blood of cynomolgus monkeys from in vivo TDAR model were measured over the 56 day study duration. Black arrows indicate placebo/KPL-404 IV administration.

\section{Figure 6: KPL-404 suppresses antigen-specific antibody responses in vivo}

Primary and secondary antigen-specific IgM responses to KLH (A) and TT (B) and IgG responses to KLH (C) and TT (D) were measured in cynomolgus monkeys from in vivo TDAR model over the 56 day study duration. These data include cohorts that received placebo/KPL-404 and KLH/TT administration on day 0 (Placebo, 1, 5 and $10 \mathrm{mg} / \mathrm{kg}$ ) and day 28 (Placebo, 1, $5 \mathrm{mg} / \mathrm{kg}$ ). ADA-positive animals were excluded from dataset as indicated in table. Black arrows indicate placebo/KPL-404 IV administration and brown arrows indicate KLH and TT antigen IM injection as appropriate. 
Figure 7: KPL-404 suppresses secondary KLH IgG recall response after prior antigen priming in the absence of KPL-404 in vivo

(A) KPL-404 serum concentrations in cynomolgus monkeys from in vivo TDAR model were measured over 28 day duration post-dose on Day 28 (B) KPL-404 CD40 receptor occupancy was measured in peripheral blood of cynomolgus monkeys from in vivo TDAR model over 28 day duration post-dose on Day 28 . (C) Primary and secondary antigen-specific IgG responses to KLH were measured in cynomolgus monkeys from in vivo TDAR model over the 56 day study duration. These data include cohorts that received KLH administration on day 0 and Placebo/KPL-404 (5 mg/kg) and KLH on day 28. Black arrows indicate Placebo/KPL-404 IV administration and brown arrows indicate KLH antigen IM injection. 


\begin{tabular}{|c|c|c|c|c|c|c|c|c|}
\hline $\begin{array}{l}\text { Group } \\
\text { (n) }\end{array}$ & $\begin{array}{l}\text { Dose } \\
\mathrm{mg} / \mathrm{kg} \\
\text { IV }\end{array}$ & Day & Gender & $\begin{array}{l}\mathrm{C}_{0} \\
\mu \mathrm{g} / \mathrm{mL} \\
\pm \mathrm{SD}\end{array}$ & $\begin{array}{l}\mathrm{C}_{\text {max }} \\
\mu \mathrm{g} / \mathrm{mL} \\
\pm \mathrm{SD}\end{array}$ & $\begin{array}{l}A C_{0-168 h r} \\
\mu g^{* h} / \mathrm{mL} \\
\pm \mathrm{SD}\end{array}$ & $\begin{array}{l}\mathrm{AUC}_{0-672 \mathrm{hr}} \\
\mu \mathrm{g}^{*} \mathrm{~h} / \mathrm{mL} \\
\pm \mathrm{SD}\end{array}$ & $\begin{array}{l}\text { HL } \\
\text { H } \\
\pm \text { SD }\end{array}$ \\
\hline $2(7)$ & 1 & 0 & $\mathrm{~F}$ & $29.3 \pm 3.33$ & $26.1 \pm 3.15$ & $1,410 \pm 302$ & $1,410 \pm 302$ & $57.7 \pm 9.29$ \\
\hline $3(7)$ & 5 & 0 & $\mathrm{~F}$ & $152^{\mathrm{a}} \pm 26.8$ & $138 \pm 15.7$ & $10,300 \pm 1,130$ & $18,500 \pm 3,860$ & $112 \pm 47.4$ \\
\hline $4(7)$ & 10 & 0 & $\mathrm{~F}$ & $303 \pm 55.3$ & $273 \pm 40.8$ & $21,900 \pm 2,010$ & $47,700^{a} \pm 5,870$ & $94.0 \pm 36.6$ \\
\hline $2(7)$ & 1 & 28 & $\mathrm{~F}$ & NR & $5.73 \pm 4.15$ & NR & NR & $\mathrm{NC}$ \\
\hline $3(7)$ & $5^{b}$ & 28 & $\mathrm{~F}$ & $145 \pm 102$ & $101 \pm 38.3$ & $16,200 \pm 3,400$ & $28,000 \pm 5,640$ & $\mathrm{NC}$ \\
\hline \multirow[t]{2}{*}{$6(7)$} & \multirow[t]{2}{*}{$5^{b}$} & \multirow[t]{2}{*}{28} & $\mathrm{~F}$ & $142 \pm 26.6$ & $158 \pm 65$ & $10,700 \pm 1,300$ & $20,900 \pm 3,900$ & $149 \pm 87.6$ \\
\hline & & & $\mathrm{M}$ & $167 \pm 15.4$ & $141 \pm 11$ & $10,600 \pm 1,050$ & $20,100 \pm 4,970$ & $109 \pm 85$ \\
\hline \multicolumn{9}{|c|}{$\begin{array}{l}{ }^{a} \text { For Group } 4 \text { receiving } 10 \mathrm{mg} / \mathrm{kg} \text { KPL-404, } \mathrm{AUC}_{0-1344 \mathrm{~h}}(\mathrm{AUC} \text { to Day 56) was } 50900000 \mathrm{ng} * \mathrm{~h} / \mathrm{mL} \\
{ }^{b} \text { Group } 3 \text { received } 5 \mathrm{mg} / \mathrm{kg} \text { KPL-404 on Days } 0 \text { and } 28 \text { and Group } 6 \text { received KPL-404 on Day } 28 \text { only } \\
\mathrm{NC}=\text { not calculated; NR = not reportable; F = Female; } \mathrm{M}=\text { Male; } \mathrm{SD}=\text { Standard Deviation }\end{array}$} \\
\hline
\end{tabular}


Figure 1

\section{Timepoints for collection of serum/blood samples}

$\downarrow$ PK $\downarrow$ RO $\downarrow$ Anti-KLH/TT $\downarrow$ ADA

Placebo/KPL-404 (i.v.)

Antigen (i.m. 3h later)

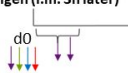

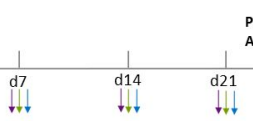

Placebo/KPL-404 (i.v.) Antigen (i.m. $3 \mathrm{~h}$ later) $n=7$ animals/group

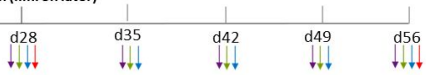

\begin{tabular}{|l|l|l|l|l|}
\hline Group & Test article & Dosing schedule (i.v.) & Antigen challenge & Dosing schedule (i.m.) \\
\hline 1 & Placebo & Day 0 and Day 28 & KLH + TT & Day 0 and Day 28 \\
\hline 2 & $1 \mathrm{mg} / \mathrm{kg} \mathrm{KPL-404}$ & Day 0 and Day 28 & $\mathrm{KLH}+\mathrm{TT}$ & Day 0 and Day 28 \\
\hline 3 & $5 \mathrm{mg} / \mathrm{kg} \mathrm{KPL-404}$ & Day 0 and Day 28 & $\mathrm{KLH}+\mathrm{TT}$ & Day 0 and Day 28 \\
\hline 4 & $10 \mathrm{mg} / \mathrm{kg} \mathrm{KPL-404}$ & Day 0 & KLH + TT & Day 0 \\
\hline 5 & Placebo & Day 28 & KLH & Day 0 and Day 28 \\
\hline 6 & $5 \mathrm{mg} / \mathrm{kg} \mathrm{KPL-404}$ & Day 28 & KLH & Day 0 and Day 28 \\
\hline
\end{tabular}


Figure 2

A.

\begin{tabular}{|l|l|l|}
\hline Antibody & Antigen & $\mathbf{K}_{\mathbf{D}}(\mathbf{n M})$ \\
\hline KPL-404 & Human CD40 & $7.2 \pm 0.3$ \\
\hline KPL-404 & Cyno CD40 & $2.9 \pm 0.05$ \\
\hline
\end{tabular}

Binding Kinetics with huCD 40 at $20 \mu \mathrm{g} / \mathrm{mL}$ Surface Density:
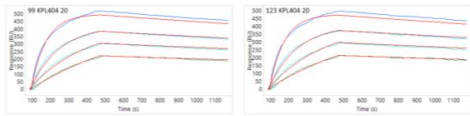

Binding Kinetics with cyCD 40 at $20 \mu \mathrm{g} / \mathrm{mL}$ Surface Density:

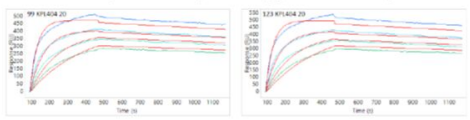

B.

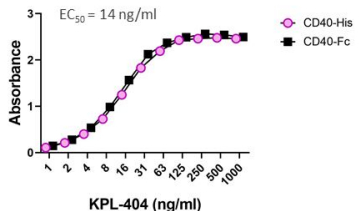

C.

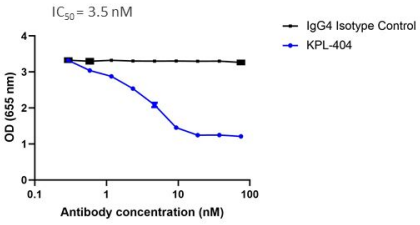




\section{Figure 3}

A. KPL-404 binding on Raji cells
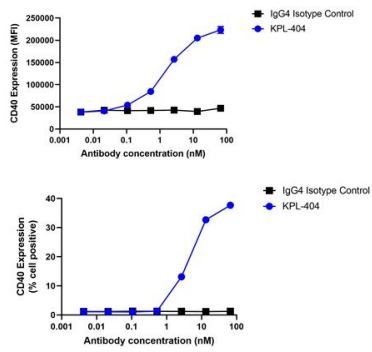

B.

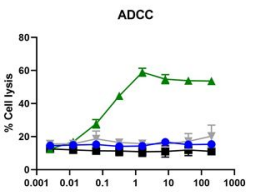

Antibody concentration (nM)

C.

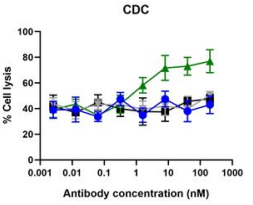

- IgG4 Isotype Control

- KPL-404

* IgG1 Isotype Control

+ Anti-CD20 (lgG1)
- IgG4 Isotype Control

- KPL-404

- I IgG1 Isotype Control

^ Anti-CD20 (lgG1) 


\section{Figure 4}

B.
A.
KPL-404 PK

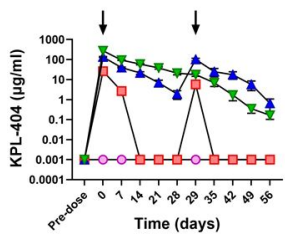

Placebo/KPL-404

IV administration

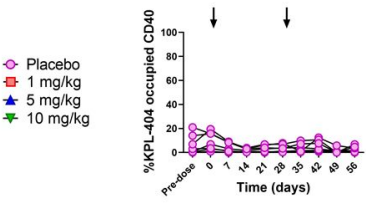

Placebo

$5 \mathrm{mg} / \mathrm{kg}$

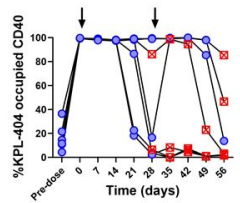

KPL-404 RO

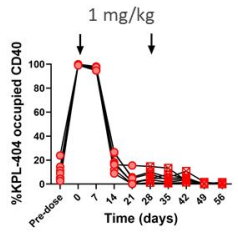

$10 \mathrm{mg} / \mathrm{kg}$

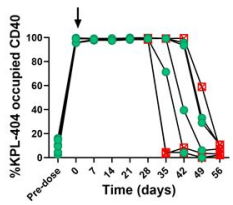

ADA positive in red crossed squares 
Figure 5

Placebo/KPL-404

$\checkmark$ IV administration

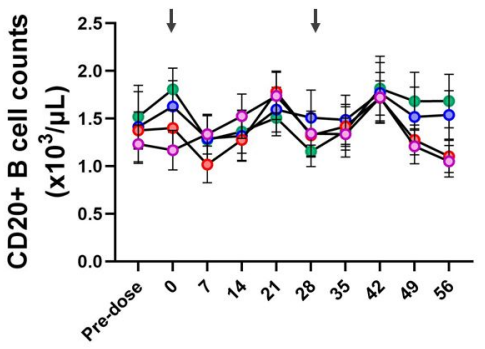

- - Placebo

$\propto 1 \mathrm{mg} / \mathrm{kg}$

- $-5 \mathrm{mg} / \mathrm{kg}$

- $10 \mathrm{mg} / \mathrm{kg}$ 
Figure 6

A. Anti-KLH IgM responses

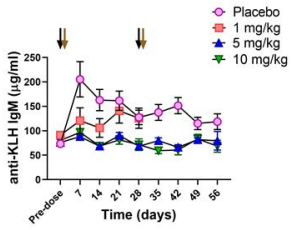

D. Anti-TTIgG responses

Placebo/KPL-404

IV administration

KLH and TT antigen IM injection

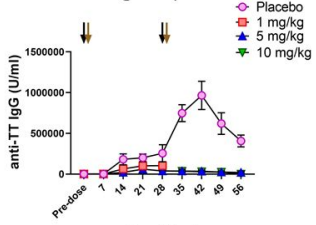

B. Anti-TT IgM responses

a. Placebo

들 $1 \mathrm{mg} / \mathrm{kg}$

t $5 \mathrm{mg} / \mathrm{kg}$

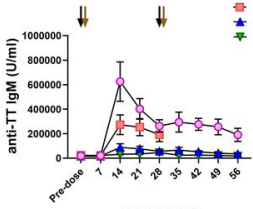

Time (days)
C. Anti-KLH IgG responses

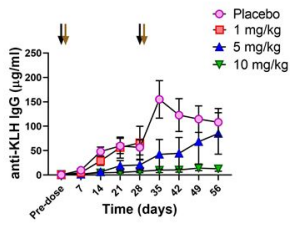


Figure 7

A.

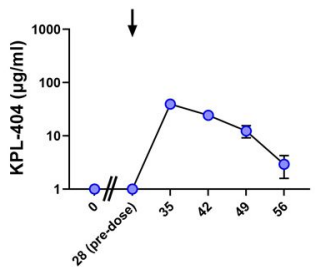

Time (days)
B.

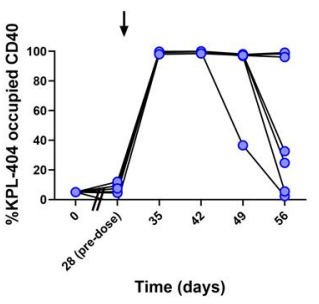

C.

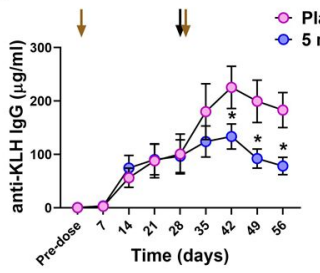

